http://doi.org/10.15359/ree.14-2.3

[Cierre de edición 15 de diciembre del 2010]

\title{
El espíritu creativo: una experiencia de investigación en educación superior
}

\section{The Creative Spirit: a Research Experience in Higher Education}

\author{
Milagro Piñeiro Ruiz ${ }^{1}$ \\ Departamento de Ciencias de la Educación \\ Sede de Occidente, Universidad de Costa Rica \\ San Ramón, Costa Rica \\ milapineiro@gmail.com, \\ milagro.pineiro@ucr.ac.cr
}

Recibido 21 de julio de $2010 \bullet$ Aceptado 31 de agosto de 2010

Resumen. El siguiente artículo presenta una experiencia de investigación desarrollada durante el I ciclo de 2009 con los estudiantes del curso Didáctica general, en la Sede de Occidente de la Universidad de Costa Rica. Esta experiencia pretende promover la creatividad de los estudiantes para la creación de su propia didáctica, a través del desarrollo de dos objetivos del curso: analizar la acción didáctica desde diferentes enfoques y determinar la relación de la didáctica con las innovaciones educativas y su vinculación con la cultura profesional. Como estrategia para la evaluación de la experiencia se utilizó la evaluación diagnóstica, formativa y sumativa.

Palabras clave. Experiencia de investigación, creatividad, didáctica, evaluación diagnóstica, evaluación formativa, evaluación sumativa, diario de triple entrada, grupo focal, taller.

Abstract. This article presents a research experience carried out during the first 2009 period with students of the General Didactic course at the Universidad de Costa Rica, Sede de Occidente. This experience intends to motivate students' creativity for the construction of their own didactic, through the development of two course objectives: to analyze didactic action from different approaches and to determine the relationship between didactic and educational innovations, and its association with professional culture. Diagnostic, formative and summative evaluations were used as a strategy to assess the experience.

Key words. Research experience, creativity, didactic, diagnostic evaluation, formative evaluation, summative evaluation, triple analysis paper, focal group, workshop.

\footnotetext{
Licenciada en la Enseñanza del Inglés y Magíster en Educación con Énfasis en Administración Educativa, ambos de la Universidad de Costa Rica. Actualmente labora como profesora en el Departamento de Ciencias de la Educación en la Sede de Occidente, Universidad de Costa Rica, impartiendo los cursos de Didáctica general, Didáctica del inglés, Tecnología educativa, Procesos en la enseñanza y aprendizaje del Inglés y Fundamentos de Administración. Encargada de la Sección de Secundaria. Investigadora de la Universidad de Costa Rica, y de la Coordinación Educativa y Cultural Centroamericana y de República Dominicana, CECC/SICA.
} 
Nuestra tarea como educadores no es la de reconocer el talento creativo posteriormente a su expresión, pero sí la de estimular el talento cuando es aún potencial y ofrecer condiciones que van a facilitar su desarrollo y expresión.

MacKinnon, 1959 (en Soriano, 2009)

\section{Introducción}

En este artículo se expone el planeamiento y desarrollo de una experiencia didáctica denominada "experiencia de investigación" la cual fue implementada con los estudiantes de las carreras de educación preescolar, primaria y secundaria en el curso ED-0012 Didáctica general, impartido en el I ciclo de 2009 en la Sede de Occidente de la Universidad de Costa Rica. Esta técnica pretende promover la creatividad de los estudiantes del curso de manera que propongan su propia didáctica a partir de las dimensiones ontológica, epistemológica, metodológica, ética y política. La técnica mencionada será abordada para el desarrollo de dos objetivos específicos: el primero, analizar la acción didáctica desde diferentes enfoques y; el segundo, determinar la relación de la didáctica con las innovaciones educativas y su vinculación con la cultura profesional. El desarrollo de la experiencia es valorada mediante la evaluación diagnóstica, formativa y sumativa. El proceso de evaluación se realiza con el fin de conocer, como lo señala Bertoni, Poggi y Teobaldo (1995), el nivel del impacto del trabajo desarrollado, a la vez que reconoce y valora los errores como fuentes de conocimiento. En esta experiencia se observa la acción tanto del estudiante como del docente y se identifican los resultados previstos y los no previstos, lo observable y no observable, con el propósito de emprender futuras acciones.

Debido a las características propias del curso, se tomaron en cuenta aspectos tales como los intereses de los estudiantes, que responden en particular a la carrera que cursan; los estilos de aprendizaje y las lecturas desarrolladas en el curso, base para generar nuevos conocimientos.

La profesora del curso Didáctica general, consciente de los cambios significativos que enfrenta el mundo en que vivimos y en particular la sociedad costarricense, además de la importancia de contribuir a la educación de los futuros profesionales en este campo, plantea, por medio del curso, el desarrollo de la experiencia didáctica como una "experiencia de investigación". Se realiza un abordaje de la didáctica desde una concepción teórico y práctica en la que se estudia la relación de la didáctica con la pedagogía, la educación y el currículo. Se pretende que el estudiante, desde diferentes concepciones -didáctica tradicional, activa y problemática- analice la necesidad de conocer e investigar aspectos relacionados con sus fundamentos, enfoques, teorías, estructura y planificación del proceso didáctico, así también el rol que juega el docente en los procesos de enseñanza y aprendizaje. Lo anterior en relación con la investigación, la innovación y los procesos de mediación que promueven la transformación de los conocimientos. De esta manera se promueve la creatividad, la cual, de acuerdo con Goleman (2000, p. 23), se define como:

(...) una musa que equivale a las buenas -y a veces geniales- ideas. El espíritu creativo es más que una iluminación ocasional o un suceso caprichoso. Cuando se despierta, el espíritu creativo anima un estilo de ser: una vida llena del deseo de innovar, de explorar nuevas formas de hacer las cosas, de convertir sueños en realidad. 
Para este autor, los momentos creativos son de vital importancia para todo lo que hacemos en cualquier aspecto de la vida, tal es el caso de las relaciones, familia, trabajo y comunidad. Sin embargo, cabe ampliar esta concepción de creatividad con lo expuesto por Betancourt (1999) cuando se refiere a la creatividad como:

El potencial humano integrado por componentes cognitivos, afectivos, intelectuales y volitivos, que a través de una atmósfera creativa se pone de manifiesto, para generar productos novedosos y de gran valor social y comunicarlos trascendiendo en determinados momentos el contexto histórico social en el que se vive. (p. 1)

La forma en que Betancourt concibe la creatividad, enfocada en el proceso de enseñanza y aprendizaje, evidencia una interrelación dialéctica que involucra no solo personas sino también al medio, al proceso y al producto. La creatividad no se enseña de manera directa, sino que se educa en la creatividad a través de diferentes actitudes tales como: involucrar a los estudiantes en la solución de determinados problemas, asumir conjuntamente los riesgos, propiciar una cultura de trabajo, confiar en el potencial que ellos tienen, responsabilizarlos de su propio aprendizaje, facilitar y mediar las oportunidades, contextualizar el conocimiento y habilidades de pensamiento crítico-creativo y motivarlos a buscar fuentes alternativas de lectura. Lo anterior asociado a una actitud transformadora del docente en la cual se propone romper resabios, mediante el principio de respeto y conciencia, tomando en cuenta las necesidades de los estudiantes.

Investigaciones realizadas sobre este tópico (Hallman, 1989; Parnes, 1989; Crawford, 1989; Davis y Scott, 1989; Marín, 1982; y Libeliy, 1993) evidencian, científicamente, que la creatividad se puede enseñar de manera inductiva. Es decir, las acciones que emprende el educador son una propuesta para su implementación, así se propicia en los estudiantes la capacidad de mejorar, captar y enfrentar problemas mediante su potencial ideativo en la cual el estudiante propone soluciones creativas mediante la receptividad de ideas y las suyas propias. Esto es, cuando la educación se convierte en aventura, se convierte en fantaeducación, la cual, según Frabboni (1998, p. 26): “(...) reconoce al sujeto su derecho a explorar las leyes de las proyecciones existenciales y de valores, a conocer alfabetos fantásticos e imaginarios, a pensar en el reino de lo <<posible>>".

Por lo tanto, se pretende, por medio del desarrollo de esta técnica, promover en el estudiante esa creatividad, a la que se refiere especialmente Betancourt, mediante el desarrollo de la unidad número tres del programa de curso, en la cual se abordan diferentes enfoques para el accionar didáctico del docente, a saber: globalización e interdisciplinariedad, socialización, enseñanza individual, autonomía, contexto, interculturalidad y diversidad.

Es importante aclarar que el curso Didáctica general es parte del "eje pedagógico", o conjunto de cursos que deben llevar todos los estudiantes empadronados en una carrera de Educación, ya sea a nivel de preescolar, primaria y secundaria. Este es brindado por el Departamento de Ciencias de la Educación y, por lo general, se programan 5 cursos a la vez en el primer ciclo lectivo. En ellos no se agrupan los estudiantes por carreras, sino que interactúan entre sí de manera que, a partir de las experiencias y conocimientos individuales y el estudio de enfoques, teorías y modelos propuestos por diferentes autores, tales como Piaget, Freire, Montesori, Neire, Sócrates, entre otros, tengan la posibilidad de empezar a proponer su propia didáctica a través de la creatividad que se promueve. 


\section{Experiencia de investigación}

La experiencia de investigación responde básicamente al desarrollo de los siguientes objetivos:

1. Analizar la acción didáctica desde el abordaje de diferentes enfoques.

2. Determinar la relación de la didáctica con las innovaciones educativas y su vinculación con la cultura profesional.

La "experiencia de investigación" incluye un trabajo de campo, la implementación de un taller y la elaboración de un diario de triple entrada. Estas tres actividades de mediación, contempladas dentro de la técnica didáctica incorporan una evaluación diagnóstica, formativa y sumativa. Diagnóstica, en tanto se parte del conocimiento que posee el estudiante antes de abordar un tema. Formativa, por todo el proceso que conlleva el proyecto de investigación, la elaboración del taller y la elaboración del diario de triple entrada. Y, finalmente, sumativa, debido a que la experiencia de investigación tiene un valor de $40 \%$ desglosado de la siguiente manera: $10 \%$ trabajo escrito de la investigación, 10\% exposición del estudio, 10\% elaboración e implementación del taller y un 10\% para el diario de triple entrada.

La decisión de implementar dicha técnica responde al acuerdo de los tres docentes que imparten el curso. Esta se fundamenta, principalmente, en la necesidad de que los estudiantes no continúen repitiendo técnicas, estrategias o métodos didácticos desde las concepciones propuestas por diferentes autores (Comenio, Makarenko, Shulman, Dewey, Peters, Bruner, Willman, Mattos, Stócker, Fernández, Tonone (Díaz, 2002); Marín (1982); Frabonni (1998); Kingler y Vadillo (2004); entre otros, quienes a lo largo de la historia han propuesto formas de enseñar, sino que a partir de teorías y de las experiencias de vida de los estudiantes se proponga una nueva forma de hacer las cosas que responda al momento histórico actual.

Para el buen desarrollo de la técnica, la docente trabajó con la siguiente metodología, esta se desarrollo por etapas.

\section{Primera etapa}

1. El docente forma ocho grupos, de acuerdo con el número de estudiantes matriculados en el curso. Se recomienda que sean los propios estudiantes quienes elijan a sus compañeros de manera que trabajen de acuerdo con sus necesidades e intereses.

2. A cada grupo se le asigna un tema de estudio. Para este caso se asignaron los siguientes temas:

- $\quad$ Globalización

- Interdisciplinaridad

- Socialización

- $\quad$ Enseñanza individual

- Contexto

- Interculturalidad

- $\quad$ Diversidad

- $\quad$ Autonomía

3. Se le solicita a cada grupo que busque y lea, de fuentes primarias y secundarias, información relacionada con el tema asignado. Este debe estar estrechamente relacionado con educación. 


\section{Segunda etapa:}

1. Una vez que cada grupo conoce más sobre el tema, la docente entrega y explica una guía didáctica (ver tabla1, 2, 3 y 4) en la cual se especifica con detalle el trabajo que realizarán los estudiantes. Esta consiste en 3 partes.

A. Propuesta e implementación de un trabajo de investigación. Contempla los siguientes elementos: portada, introducción, un objetivo general y al menos tres específicos, metodología, marco conceptual, marco teórico, análisis de la información, conclusiones, recomendaciones, bibliografía y anexos.

B. Exposición de la investigación. Contempla los siguientes elementos: responsabilidad, puntualidad y organización; creatividad y originalidad; uso y calidad de recursos materiales; claridad en las indicaciones; dominio del tema y trabajo en equipo.

C. Planeamiento y ejecución de un taller. Se divide en dos secciones:

- Informe escrito del planeamiento del taller: Contempla los siguientes elementos: objetivo general, objetivos específicos, contenidos, procedimientos (actividades de mediación), valores y actitudes, tiempo, evaluación, anexos (materiales, resúmenes, fichas, láminas, entre otros.)

- Implementación del taller. Considera: la responsabilidad, puntualidad y organización, creatividad y originalidad, claridad de ideas, variedad de actividades, dominio del tema, aporte de ideas, participación de los receptores, ideas para la construcción de conocimientos.

Tabla 1

Informe escrito (30 puntos)

Los profesores ofrecen la oportunidad a los estudiantes de trabajar en grupos de un máximo de cuatro personas. El trabajo tiene como objetivo investigar, elaborar y exponer, de forma vivencial, una temática asignada por los docentes del curso. El trabajo escrito comprende los criterios de evaluación que se establecen en la siguiente tabla:

\begin{tabular}{|l|c|}
\hline ASPECTO POR EVALUAR & PUNTAJE \\
\hline- Portada & 1 \\
\hline- Introducción & 1 \\
\hline- Un objetivo general y tres específicos & 2 \\
\hline- Metodología & 2 \\
\hline- Marco conceptual & 4 \\
\hline- Marco teórico & 4 \\
\hline- Análisis de la información & 3 \\
\hline- Conclusiones & 2 \\
\hline- Recomendaciones (propuesta de taller) & 2 \\
\hline- Referencias bibliográficas & 1 \\
\hline- Anexos & 4 \\
\hline- Otros criterios por evaluar: puntualidad, estética, entrega del trabajo en forma impresa & \\
\hline & y digital \\
\hline
\end{tabular}


a. Durante las horas de consulta, los estudiantes podrán evacuar dudas sobre la experiencia pedagógica. Es importante aclarar que estas horas de consulta tienen como finalidad dar seguimiento al desarrollo del trabajo, no realizar correcciones periódicas.

Tabla 2

Exposición (20 puntos)

Los estudiantes contaron con 60 minutos para exponer. Las exposiciones se realizarán según cronograma de curso. Los rubros por calificar serán los especificados en la tabla 2 que se presenta a continuación:

\begin{tabular}{|l|c|}
\hline ASPECTOS POR EVALUAR & PUNTAJE \\
\hline$-\quad$ Responsabilidad (se evalúa individualmente) & 2 \\
\hline$-\quad$ Puntualidad y organización & 2 \\
\hline- Creatividad y originalidad & 4 \\
\hline$-\quad$ Uso y calidad de recursos materiales & 3 \\
\hline- Claridad en las indicaciones & 2 \\
\hline- Dominio del tema (se evalúa individualmente) & 5 \\
\hline$-\quad$ Trabajo en equipo & 2 \\
\hline
\end{tabular}

Tabla 3

Exposición del taller (20 puntos)

Tema:

Integrantes:

\begin{tabular}{|l|c|}
\hline ASPECTOS POR EVALUAR & PUNTAJE \\
\hline$-\quad$ Responsabilidad (se evalúa individualmente) & 2 \\
\hline- Puntualidad y organización & 2 \\
\hline$-\quad$ Creatividad y originalidad & 2 \\
\hline$-\quad$ Claridad de ideas & 2 \\
\hline$-\quad$ Variedad de actividades & 4 \\
\hline$-\quad$ Dominio del tema & 4 \\
\hline$-\quad$ Aporte de ideas para la construcción de conocimientos & 2 \\
\hline$-\quad$ Participación de los receptores & 2 \\
\hline
\end{tabular}


Tabla 4

Informe escrito del planeamiento del taller (15 puntos)

\begin{tabular}{|l|c|}
\hline ASPECTOS POR EVALUAR & PUNTAJE \\
\hline- Objetivo general & 1 \\
\hline- Objetivos específicos (mínimo 3) & 3 \\
\hline- Contenidos & 1 \\
\hline- Procedimientos (actividades de mediación) & 6 \\
\hline$-\quad$ Valores y actitudes & 1 \\
\hline$-\quad$ Tiempo & 1 \\
\hline$-\quad$ Evaluación & 1 \\
\hline$-\quad$ Anexos (materiales, resúmenes, fichas, láminas, entre otros.) & 1 \\
\hline
\end{tabular}

Nota. Los puntos contemplados en el informe escrito del planeamiento del taller, únicamente se obtendrán si este ha sido revisado y aprobado por el profesor de curso, con ocho días de anticipación. Se debe presentar el borrador y las correcciones del planeamiento el día de su realización.

\section{Tercera etapa:}

\section{Asignación de "Diario de triple entrada"}

A la luz de los temas estudiados, los estudiantes retoman los enfoques didácticos (tradicional, activo y problemático) y realiza un Diario de triple entrada (ver tabla 5). Este consta de tres columnas:

- En la primera, el estudiante debe escribir, mediante una cita bibliográfica, la definición de cada uno de los enfoques estudiados.

- $\quad$ En la segunda, realiza la interpretación y análisis de cada una de las definiciones de los enfoques a la luz de las exposiciones de sus compañeros, sus experiencias vividas y el ambiente que lo rodea.

- En la tercera, debe explicar para cada uno de los enfoques, como se ven reflejados en la película "Los escritores de la libertad", la cual está basada en una historia de la vida real que vive un grupo de estudiantes y su profesora de curso.

- Seguido de las columnas incorporará la bibliografía consultada. 
Tabla 5

Diario de triple entrada

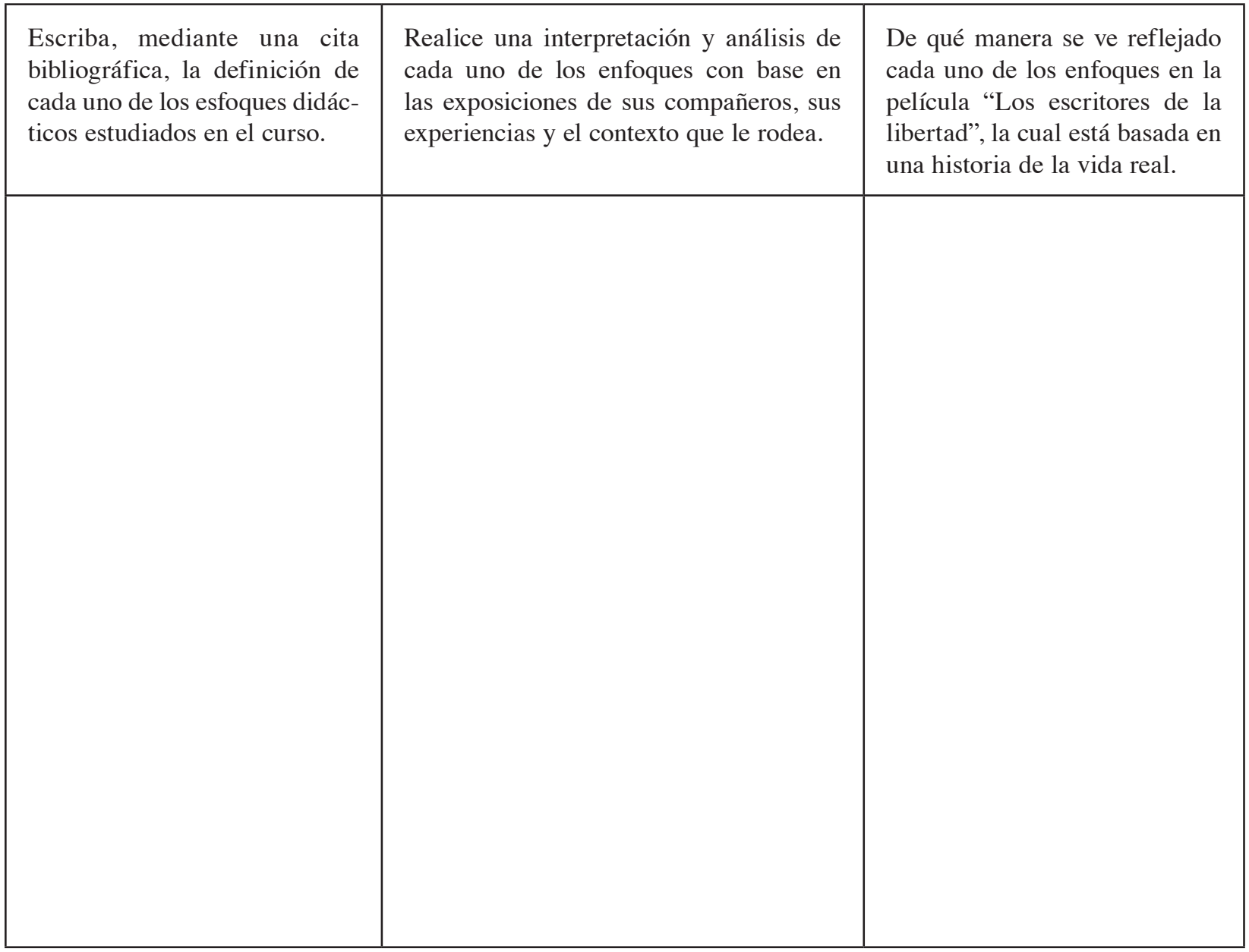

Nota. Este es el formato que brinda la docente; sin embargo, puede ser cambiado, a comodidad del estudiante, siempre y cuando incluya toda la información que se le solicita de manera clara y ordenada.

\section{Papel del docente en la estrategia}

La participación del docente como guía en la planificación e implementación de la técnica didáctica, así como el diario de triple entrada se fundamenta en los principios de la didáctica problemática en la cual, según Vadillo y Kingler (2004), el estudiante debe ir más allá del conocimiento, se relacionan todas las partes de su ser: lo cognitivo, la ética, y la estética, las cuales se sustentan en las corrientes del constructivismo -en cuanto el estudiante tiene la posibilidad de participar en la construcción de su propio conocimiento-, lo cual le proporciona otras habilidades como trabajar de manera independiente, investigar de acuerdo con su nivel y crecer y desarrollarse cognitivamente, a la vez que se responsabiliza frente a los problemas que enfrenta la educación de manera que busque justicia.

La "experiencia de investigación" se realiza durante tres meses, mientras atiende el horario del curso, además cuenta con un periodo de 4 horas semanales destinadas por la docente a la atención de horas estudiante (HAE). En las HAE los discentes pueden evacuar dudas, externar situaciones 
generales con respecto al trabajo y llevar avances del mismo para ser revisados por el docente. Este proceso se enfoca en la evaluación formativa.

Además, el docente, por medio de una guía de evaluación (ver tabla 2, 3, 4 y 5), realiza una evaluación sumativa del trabajo.

\section{Papel de estudiante en la estrategia}

El estudiante es el protagonista principal en la planificación y desarrollo de la estrategia, es a quien le corresponde investigar, sistematizar, planificar y desarrollar la experiencia de investigación. Todo esto con la guía y apoyo del profesor de curso.

\section{Papel de contexto en la experiencia}

Para el desarrollo de la experiencia de investigación, el contexto en el cual está inmerso el estudiante universitario juega un papel muy importante. Para este trabajo se seguirá lo expuesto por Wang (1994), quien se refiere al contexto como el entorno lingüístico y físico del cual depende el sentido y valor de una palabra, frase o fragmento, así como la situación, ya sea política, histórica, cultural o de cualquier otra índole, en la cual se considera un hecho.

En primer lugar, para el caso particular de los estudiantes matriculados en el curso, el contar con la biblioteca Carlos Monge Alfaro permite el acceso a gran cantidad de recursos impresos y digitales, así como salas de estudio y de reuniones. En segundo, la Sede de Occidente cuenta no solo con un laboratorio informático con acceso a internet, el cual pueden utilizar sin ningún costo, sino que también se habilitó una red inalámbrica, de manera que todos aquellos que cuenten con computadora pueden consultar, enviar y recibir documentos desde sus propios equipos, lo que propicia un contacto directo con la profesora del curso; por medio del internet. En tercer lugar, la Sede cuenta con un servicio de fotocopiadora dentro de instalaciones, así se facilita la impresión de documentos requerdos. En cuarto lugar, los grupos quedaron integrados por estudiantes de diferentes carreras, por lo que el aporte que cada uno de ellos, desde su campo de estudio, es de gran importancia para el desarrollo de la técnica. En quinto lugar, en los alrededores de la Universidad existen varias instituciones educativas públicas, por lo que los estudiantes que así lo desearan, tenían la posibilidad de desarrollar la investigación en estas. Se propicia, de esta manera, que se enfrenten, desde los primeros años de carrera, a situaciones reales vividas por las personas en estos centros de estudio. Finalmente, la profesora del curso cuenta con una oficina dentro del campus para atender a los estudiantes personalmente, en caso de que estos así lo requieran, lo que representa una guía constante para el éxito de la experiencia.

\section{Aplicación de la experiencia}

La técnica fue aplicada en el curso Didáctica General. Se asignó el día lunes 9 de marzo, día de inicio de lecciones, y concluyó el lunes 22 de junio de 2009, fecha en la que se entregó el último trabajo, "Diario de triple entrada", por parte de los estudiantes. Es importante aclarar que el curso contó con una matrícula de 40 estudiantes, con un horario de 6 horas semanales: 4 de teoría y 2 de 
práctica. Fue impartido en el aula 205, segundo pabellón, de la Sede de Occidente de la Universidad de Costa Rica.

Al ser un curso presencial, la comunicación fue constante para el buen desarrollo de la experiencia. Esta propició el análisis y vivencia de situaciones reales a las que se enfrentará el estudiante, como educador, en un futuro próximo, de manera que tanto la investigación, el taller y el diario de triple entrada permitió a los educandos, a partir de esas vivencias, desarrollar una lección para el abordaje del tema en estudio.

\section{Evaluación de la técnica}

Dentro de las técnicas seleccionadas para evaluar la experiencia de investigación, se utilizó, en primera instancia, la evaluación diagnóstica, la cual fue aplicada por el docente, para conocer qué tanto sabían los estudiantes sobre los diferentes enfoques de la didáctica y la relación de esta con las innovaciones educativas y su vinculación con la cultura profesional.

Para asignar una calificación al trabajo desarrollado por los estudiantes, se recurrió, en segunda instancia, a la evaluación sumativa. Es decir, a cada trabajo desarrollado por el estudiante se le asignó un valor numérico desglosado de la siguiente manera: 10\% al trabajo escrito, 10\% a la exposición y $10 \%$ al taller (ver tabla 1).

Finalmente, y la más importante, se evaluó la experiencia por medio de la evaluación formativa. Esta se dividió en dos etapas. Primero, se dio seguimiento a los estudiantes al planear, implementar y evaluar el trabajo realizado y; segundo, se realizó la técnica de "grupo focal", la cual le permitió, a la profesora de curso, evaluar el desarrollo de la experiencia mediante la interacción colectiva de estudiantes. Para este caso en particular, se trabajó el último día de lecciones con solo diez estudiantes escogidos al azar, siguiendo dos procedimientos:

- Se invitó a 10 estudiantes a participar voluntariamente, a lo que todos aceptaron.

- Se les explicó la dinámica de la técnica. Esta consistió en que los estudiantes respondieran a una única pregunta: ¿Qué les pareció la experiencia de investigación? Mientras los estudiantes daban a conocer sus percepciones, la docente tomaba apuntes y guiaba el proceso de manera que no se salieran del tema.

\section{Conclusiones}

Una vez finalizada la experiencia de investigación se llega a las siguientes conclusiones:

- $\quad$ Por medio de la experiencia de investigación, los estudiantes tuvieron la posibilidad de realizar un abordaje creativo de la "didáctica" desde la concepción problemática, sin dejar de lado los fundamentos epistemológicos y ontológicos de la didáctica tradicional y activa. Lo anterior permitió una interrelación dialéctica que involucró personas, medio, proceso y producto, tal y como lo establece Betancourt (1999), al afirmar que estos procesos facilitan el generar productos novedosos y de gran valor social. 
- $\quad$ Por medio de la estrategia y según el enfoque asignado, los estudiantes se enfrentaron a situaciones reales que viven docentes y estudiantes en las diferentes instituciones educativas de la región de occidente (Colegio Experimental Bilingüe de Palmares, Naranjo y San Ramón, Escuela Jorge Washington en San Ramón, Kinder Central Valverde Vega, entre otros) A partir de ello, se busca proponer soluciones desde su percepción, potencial intelectual y formación académica.

- Tal y como lo establece Goleman (2000) cuando se refiere a la creatividad como el deseo de innovar, de explorar nuevas formas de hacer las cosas y de convertir sueños en realidad, por medio del trabajo cooperativo e interdisciplinario que realizó cada grupo, se evidenciaron, en los estudiantes, habilidades propias del pensamiento crítico creativo y potencial ideativo, al proponer soluciones creativas en conjunto, de acuerdo con situaciones reales vividas en los salones de clase. Esto, a su vez, es conocido por Frabboni (1998) como fantaeducación, la cual nace de lo que sé y de lo que aprendo para motivar a nuevas formas de hacer las cosas.

- $\quad$ Algunos factores asociados con el desarrollo de la experiencia de investigación tales como: conocimiento en el campo de la investigación, motivación, preparación para enfrentarse a situaciones nuevas, seguimiento oportuno y disponibilidad de tiempo, por parte de la docente del curso de Didáctica general, fueron claves para influir positivamente en el desarrollo del espíritu creativo que se pretendió y consiguió en los estudiantes del curso. Esta posición es apoyada por Soriano (2009) cuando afirma que la creatividad puede ser enseñada o aprendida siempre y cuando propicie en el estudiante una actitud de compromiso ante los desafíos que se le propongan.

- El que los estudiantes tengan la posibilidad de evaluar las mediaciones pedagógicas implementadas por los docentes, para este caso por medio de un "grupo focal" al final de la experiencia de investigación, permitió a la docente conocer no solo el nivel de aceptación de la estrategia, sino la necesidad de realizar algunos cambios que mejoren estos procesos utilizados a nivel universitario. Importante resaltar algunas apreciaciones de los estudiantes en torno a esta experiencia en sí:

- Los estudiantes participantes coincidieron en que el desarrollo de este trabajo resultó muy provecho, pues les permitió, una vez conocidos y analizados los diferentes enfoques, teorías y modelos de la didáctica, proponer y desarrollar la propia.

- $\quad$ Ellos agregan que el desarrollo de la experiencia se dio a través de su propia creatividad y del principio de que se aprende haciendo, sin dejar de lado las dimensiones ontológica, epistemológica, metodológica, ética y política que conlleva desarrollar una experiencia de investigación de este tipo.

- Afirman que el seguimiento, por parte de la docente, para llevar a cabo la experiencia, facilitó el comprender situaciones reales que enfrentan los docentes en ejercicio, lo cual permite no solo valorar la necesidad de desarrollar nuevas habilidades y estrategias de trabajo en las instituciones educativas, sino también el des-aprendizaje de una serie de actitudes que llevan a la persona y especialmente al educador a actuar sin sentido.

- Por último, los estudiantes fueron enfáticos en indicar que, en especial, el taller y el diario de triple entrada les ofreció un espacio para la reflexión sobre el papel que 
realiza y debería realizar el docente, de acuerdo con las situaciones que enfrenta durante el proceso de enseñanza y aprendizaje y de acuerdo con el contexto social, político, cultural e histórico en que se encuentra.

\section{Limitaciones encontradas}

Las limitaciones en el desarrollo de la experiencia estuvieron marcadas por tres condiciones básicamente:

- Desinterés mostrado por tres estudiantes, quienes expresaron abiertamente estar empadronados en una carrera de educación que no les gusta, pues el promedio no les dio para ingresar a la carrera de Derecho. Esta condición se reflejó directamente en el desarrollo de la experiencia, básicamente por el desinterés hacia el trabajo y el poco aporte que dieron.

- Conflictos entre compañeros por el nivel de compromiso y responsabilidad que cada uno asume.

- $\quad$ Problemas de redacción y ortografía en el trabajo escrito, situación que dificulta tener una lectura fluida y comprensible del trabajo realizado.

\section{Referencias bibliográficas}

Bertoni, A., Poggi, M y Teobaldo, M. (1995). Evaluación de nuevos significados para una práctica compleja. Buenos Aires: Editorial Kapeluz.

Betancourt, J. (1999, Julio-Septiembre). Creatividad en la educación: Educar para transformar. Revista Educar, Número 10, s. p. Recuperado de http://educar.jalisco.gob.mx/10/10julian. $\underline{\text { html }}$

Betancourt, J. (2000). Creatividad en la educación: Educación para transformar. Revista Electrónica Psicología Científica.com. Recuperado de http://www.psicologiacientifica.com/bv/psicologia183-1-creatividad-en-la-educacion-educacion-para-transformar.html.

Crawford, R. (1989). Las técnicas de la creatividad. En G. Davis \& J. Scott (Orgs.). Estrategias para la creatividad, (pp. 7-12). México: Paidós Educador.

Díaz, F. (2002). Didáctica y Currículum: un enfoque constructivista. Cuenca: Ediciones de la Universidad de Castilla.

Frabboni, F. (1998). El libro de la pedagogía y la didáctica I. La educación. Madrid: Editorial Popular.

Goleman, D. (2000). El espíritu creativo. Madrid: Vergara. 
Hallman, R. (1989). Técnicas de enseñanza creativa. En G. Davis \& J. Scott (Orgs.). Estrategias para la creatividad, (pp. 12-13). México: Paidós Educador.

Libeliy, P. (1993). Creatividad y Universidad, ¿Qué tal pareja son? DIDAC. Reflexiones sobre la educación. 2 J. Primavera' 93. Consultada en http://www.uia.mx/web/site/tpl-Nivel2.php?men $\underline{\mathrm{u}}=\mathrm{mg}$ Publicaciones\&seccion $=$ puDidac

Marín, I. (1982). Principios de la educación contemporánea. Madrid: Rialp.

Parnes, S. (1989). ¿Puede incrementarse la creatividad? G. Davis y J. Scott (Orgs.). Estrategias para la creatividad, (pp. 8-9). México: Paidós Educador.

Soriano, E. (2009, Julio-Septiembre). La educación para la creatividad. Revista Educar,

Vadillo, G. y Kingler, C. (2004). Didáctica. Teoría y práctica de éxito en Latinoamérica y España. México: Editorial Mc Graw Hill.

Wang, M. (1994). Atención a la diversidad del alumnado. Madrid: Narcea. 